\title{
Observation of Thermal Beaming from Tungsten and Molybdenum Bull's Eyes
}

\author{
Jong Hyuk Park, ${ }^{\dagger, \xi}$ Sang Eon Han, ${ }^{\xi,+}$ Prashant Nagpal, ${ }^{\S, \#}$ and David J. Norris ${ }^{*, \dagger}$ \\ ${ }^{\dagger}$ Optical Materials Engineering Laboratory, ETH Zurich, 8092 Zurich, Switzerland \\ ${ }^{\S}$ Department of Chemical Engineering and Materials Science, University of Minnesota, \\ Minneapolis, Minnesota 55455, United States \\ TPhoto-Electronic Hybrids Research Center, Korea Institute of Science and Technology, \\ Seoul 136-791, South Korea \\ ${ }^{\ddagger}$ Department of Chemical and Biological Engineering, University of New Mexico, \\ Albuquerque, New Mexico 87131, United States \\ \#Department of Chemical and Biological Engineering, University of Colorado, \\ Boulder, Colorado 80303, United States
}

\section{Supporting Information}

\section{Fabrication of metallic bull's-eye structures}

The silicon templates for the metallic bull's-eye structures were prepared via photolithography. Positive photoresist (Shipley, Microposit S1805) was spin-coated at 4000 rpm on pre-cleaned $\mathrm{Si}$ substrates and then exposed under an ultraviolet lamp through a chrome-on-glass mask with a mask aligner (Karl Suss, MA6). The photoresist on the exposed areas was removed in a developing solution (Shipley, Microposit 351 developer:deionized water $=1: 5)$. The patterns of the photoresist layer were transferred to the underlying $\mathrm{Si}$ substrates by reactive-ion etching (RIE). The RIE process (Oxford Instrument, RIE 80 Plus) 
was performed at a power of $25 \mathrm{~W}$ and a pressure of 100 mTorr with $\mathrm{CF}_{4}$ and $\mathrm{O}_{2}$ gas flow rates of $40 \mathrm{sccm}$ and $4 \mathrm{sccm}$, respectively. The etching rate was about $0.6 \mathrm{~nm} / \mathrm{s}$ and the depth of the grooves was controlled by varying the process time.

Tungsten and molybdenum layers were deposited with dc magnetron sputtering (Kurt Lesker, PVD 75) at a power of $150 \mathrm{~W}$. The diameter of the sputtering targets was 2 inches. The pressure in the sputtering chamber was maintained at 6 mTorr with argon during deposition and the source-to-substrate distance was fixed as $13 \mathrm{~cm}$. The sputtering time for each material was controlled to obtain about 160-nm-thick layers.

\section{Deposition of a protective oxide layer on metal surfaces}

To enhance the thermal stability of metallic bull's eyes, their surfaces were coated with a $\mathrm{HfO}_{2}$ layer via atomic layer deposition (ALD, Picosun, R150B). ${ }^{1,2}$ Tetrakis(ethylmethylamido)hafnium (TEMAH, $\left.\mathrm{Hf}\left[\mathrm{N}\left(\mathrm{CH}_{3}\right)\left(\mathrm{C}_{2} \mathrm{H}_{5}\right)\right]_{4}\right)$ was utilized as a precursor and reacted with water at $200{ }^{\circ} \mathrm{C}$. TEMAH and water vapors were alternatively added to the $\mathrm{N}_{2}$ carrier flow of $100 \mathrm{sccm}$ and the pulse time for both vapors was $0.1 \mathrm{~s}$. One ALD reaction cycle was defined as one dose of the precursor followed by a 6-s purge and then one dose of water followed by an 8-s purge. An 11-nm-thick $\mathrm{HfO}_{2}$ layer was formed on the metal surfaces after 125 reaction cycles.

\section{Characterization of the surface morphology}

An atomic force microscope (AFM, Agilent 5500) was utilized to characterize the surface morphology of the metal films (see Figure S1). All AFM images were obtained by using tapping mode and the root-mean-square (RMS) roughness of the metal surfaces was determined with $2.5 \times 2.5 \mu \mathrm{m}^{2}$ scans. 


\section{Alignment of the experimental setup for thermal-emission measurement}

Since the thermal emission from the bull's eye is highly directional, the experimental setup required precise alignment to measure the emission spectrum. Figure S2 describes the setup and contains the abbreviation of each component. The alignment was done by the following procedure. (i) The position of pinhole 2 (P2) was fixed and utilized as the reference point. (ii) The red laser beam coming from the interferometer in the Fourier-transform infrared (FTIR) spectrometer (Bruker, Vertex 80) was aligned to the center of P2 by adjusting the tilt angle of the focusing mirror (FM). (iii) The center position of pinhole 1 (P1) was determined by using the laser beam. (iv) The tilt angle of the mirror (M) was then adjusted to reflect the laser beam to the center of P1. (v) M is turned without changing the tilt angle to reflect the laser beam to the center of the heating chamber (HC). (vi) The position of pinhole 3 (P3) was determined by using the laser beam. (vii) The sample was placed on the center of HC. (viii) The tilt angle of the HC was adjusted by using a goniometer to align the reflected laser beam from the sample to the center of P1. (ix) The size of the hole of P3 was controlled to block the thermal emission from the surrounding regions. (x) The size of P1 and P2 was controlled to obtain a collection angle of $\pm 0.1^{\circ}$.

FM and $\mathrm{M}$ were made from silver, which is highly reflective at infrared wavelengths. In addition, a sapphire flat with a high transmittance in the infrared wavelength range was utilized as a window for $\mathrm{HC}$ to minimize the loss of thermal emission from the bull's eye.

\section{Measurement of thermal emission spectra}

For the measurement of the thermal-emission spectra, the heating chamber (Linkam, TS1500) was evacuated with a turbomolecular pump (Varian, Turbo-V 70). After the chamber pressure reached around $10^{-5}$ mbar, the sample was heated to $900{ }^{\circ} \mathrm{C}$ at a ramping rate of $20{ }^{\circ} \mathrm{C} / \mathrm{min}$. During the measurement, the temperature of the heater was constant 
$\left(900 \pm 1^{\circ} \mathrm{C}\right)$ and the outside of the chamber was kept at room temperature by cooling water.

The thermal-emission spectrum from the bull's eye was measured with an FTIR spectrometer (Bruker, Vertex 80). Due to the IR wavelength of the emission peak, a $\mathrm{KBr}$ beam splitter and a liquid-nitrogen-cooled InSb detector were utilized. The emission spectrum from the sample was scanned 4000 times in the wavenumber range between 3400 and $2400 \mathrm{~cm}^{-1}$ with a resolution of 4 or $8 \mathrm{~cm}^{-1}$. To calibrate the emissivity, we assumed that the thermal emission from a carbon emitter (Aldrich, carbon nanopowder, \#633100, which we manually pelletized) is equivalent to that from a blackbody at the given temperature. Then, the emission spectrum from the sample was normalized by that of the carbon emitter obtained under the same measurement conditions. The tilt angle of the sample could be accurately controlled by using a goniometer on which the heating chamber was mounted (see Figure S2b).

\section{Calculation of thermal emission spectra via numerical simulation}

We assumed that the size of the bull's eye is sufficiently large that we could neglect the effect of the center and the boundary of the structure on its thermal-emission properties. Hence, the bull's eye could be approximated as a series of linear gratings oriented around the

center. ${ }^{3}$ Since the absorptivity is equal to the emissivity according to Kirchhoff's law, ${ }^{4,5}$ the averaged absorptivity of linear gratings evenly distributed in all directions yields the emissivity of the bull's eye. For the calculation, we used the transfer-matrix formalism, ${ }^{6}$ and a unit cell is discretized by an $80 \times 80$ mesh. The simulation was performed for each structure using the experimental dimensions obtained from scanning electron microscopy. While all bull's eyes had nominally the same period $(3.52 \mu \mathrm{m})$ and groove depth $(180 \mathrm{~nm})$, the fill factor varied slightly. The bare $\mathrm{W}, \mathrm{HfO}_{2}$-coated $\mathrm{W}$, and $\mathrm{HfO}_{2}$-coated Mo structures had fill factors of $0.5,0.52$, and 0.54 , respectively. The thickness of the $\mathrm{HfO}_{2}$ layer was $11 \mathrm{~nm}$ and its 
dielectric constant was assumed to be 4 without an imaginary component. ${ }^{7}$ For W, the dielectric function at $900{ }^{\circ} \mathrm{C}$ was employed to obtain the emission spectrum. ${ }^{8}$ However, due to the absence of data for Mo at the elevated temperatures, the dielectric function for room temperature was assumed for the calculation. This resulted in large differences between the measured and calculated results in terms of the overall emissivity and the width of the emission peak.

\section{Supporting references}

1. Hausmann, D. M.; Kim, E.; Becker, J.; Gordon, R. G. Atomic layer deposition of hafnium and zirconium oxides using metal amide precursors. Chem. Mater. 2002, 14, 4350-4358.

2. Kukli, K.; Ritala, M.; Sajavaara, T.; Keinonen, J.; Leskelä, M. Atomic layer deposition of hafnium dioxide films from hafnium tetrakis(ethylmethylamide) and water. Chem. Vap. Deposition 2002, 8, 199-204.

3. Han, S. E.; Norris, D. J. Beaming thermal emission from hot metallic bull's eyes. Opt. Express 2010, 18, 4829-4837.

4. Greffet, J.-J.; Nieto-Vesperinas, M. Field theory for generalized bidirectional reflectivity: derivation of Helmholtz's reciprocity principle and Kirchhoff's law. J. Opt. Soc. Am. A 1998, 15, 2735-2744.

5. Han, S. E. Theory of thermal emission from periodic structures. Phys. Rev. B 2009, 80, 155108.

6. Pendry, J. B.; MacKinnon, A. Calculation of photon dispersion relations. Phys. Rev. Lett. 1992, 69, 2772-2775.

7. Triyoso, D. H.; Hegde, R. I.; White, J. B. E.; Tobin, P. J. Physical and electrical characteristics of atomic-layer-deposited hafnium dioxide formed using hafnium tetrachloride and tetrakis(ethylmethylaminohafnium). J. Appl. Phys. 2005, 97, 124107.

8. Aksyutov, L. N. Temperature dependence of the optical constants of tungsten and gold. J. Appl. Spectrosc. 1977, 26, 656-660. 


\section{Supporting Figures}
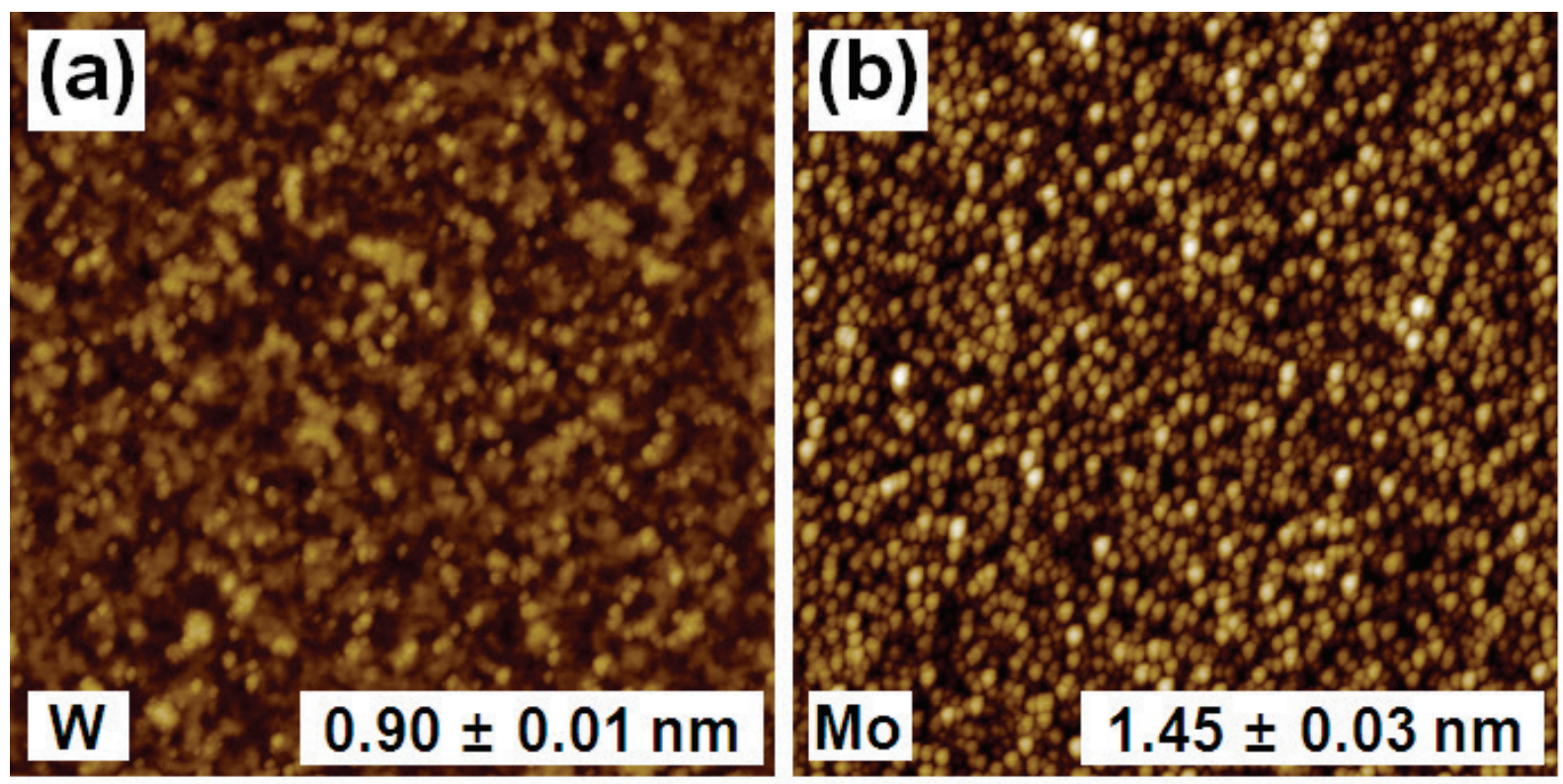

$0 \mathrm{~nm}$

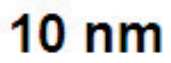

Figure S1. Atomic force micrographs of our (a) tungsten (W) and (b) molybdenum (Mo) films deposited on silicon substrates. Each image includes the extracted RMS roughness from the $2.5 \times 2.5 \mu \mathrm{m}^{2}$ scanned area. 

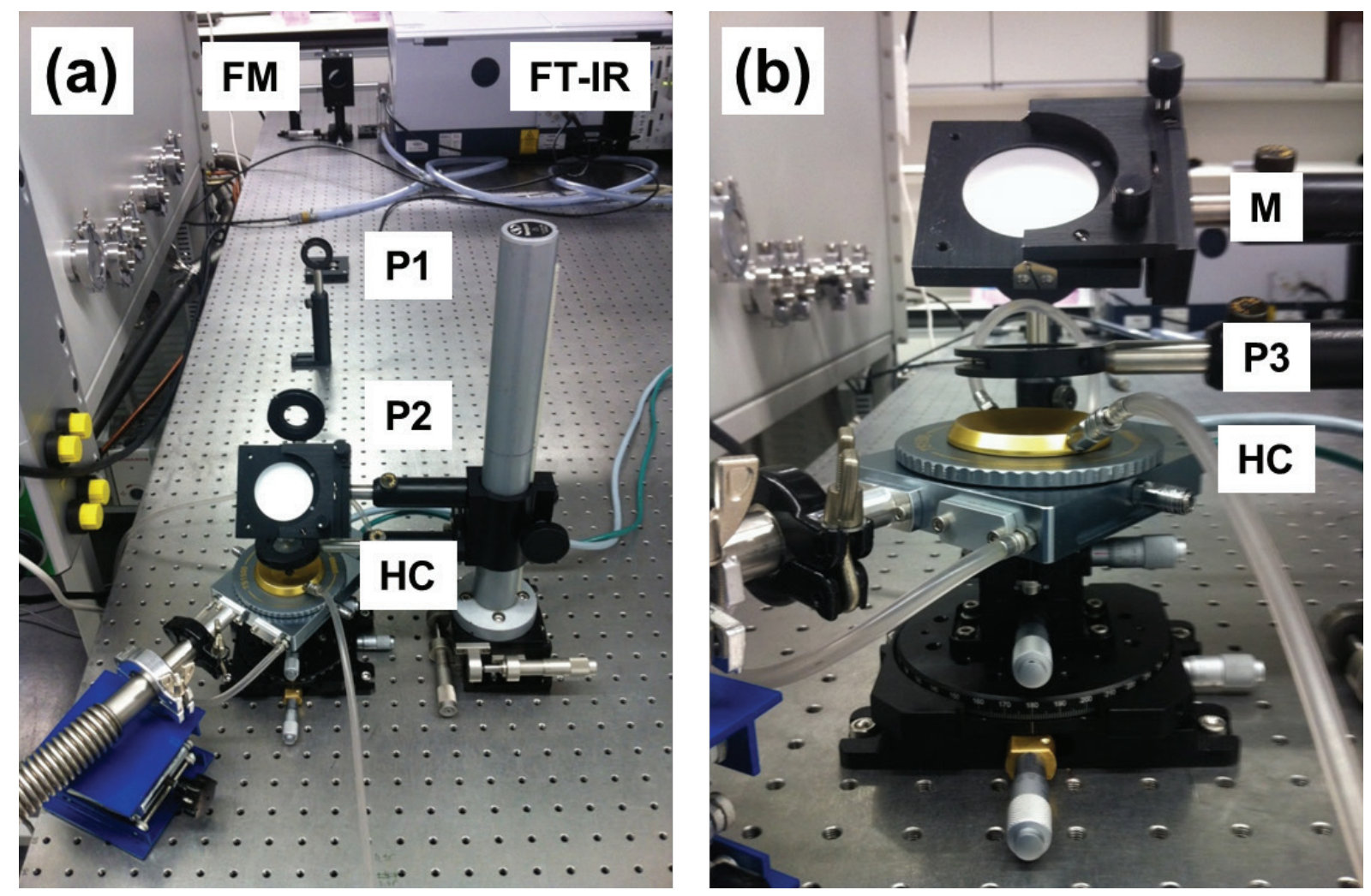

Figure S2. Photographs of the experimental setup for measuring thermal-emission spectra from bull's eyes. The heating chamber is connected to a turbomolecular pump for evacuation and the outside of the chamber is cooled to room temperature by water circulation. The abbreviation of each component is as follows: mirror (M); focusing mirror (FM); pinhole 1, 2, and 3 (P1, P2, and P3, respectively); and heating chamber (HC). 

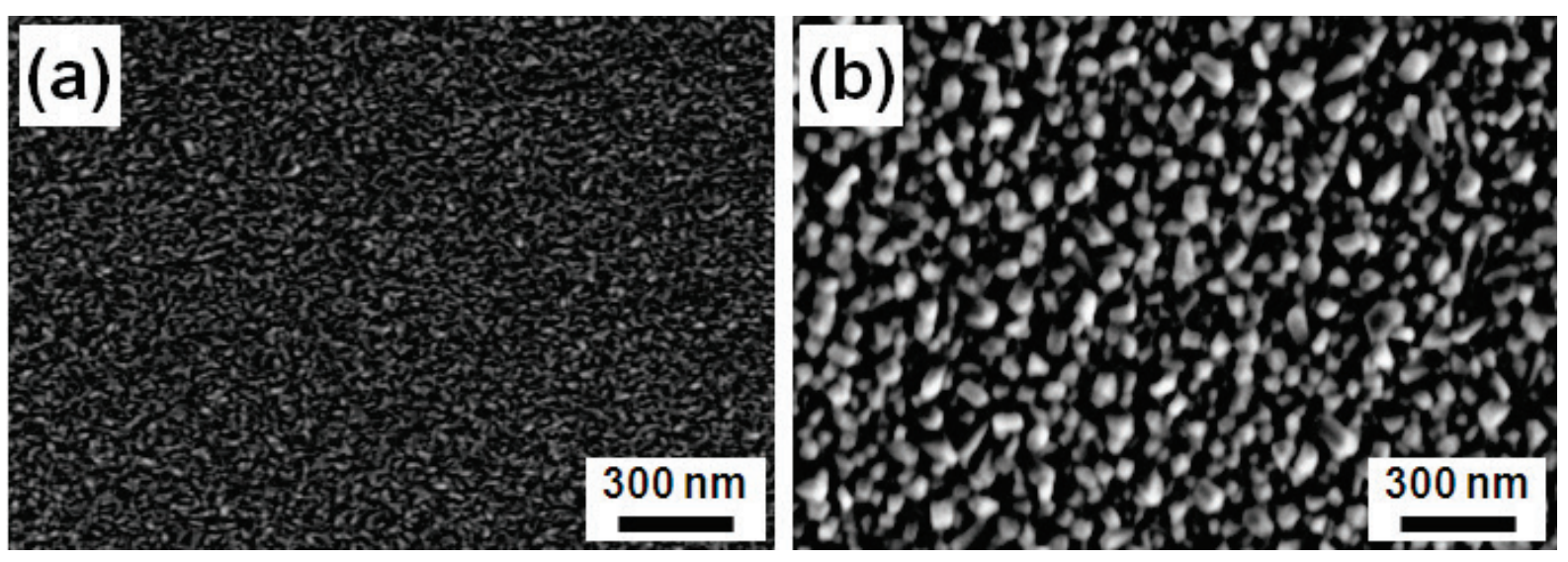

Figure S3. Scanning electron micrographs of a molybdenum bull's eye before and after heating. The surface of a ridge in the bull's eye (a) before and (b) after $2 \mathrm{~h}$ at $600{ }^{\circ} \mathrm{C}$. The surface morphology is changed significantly after heating. 